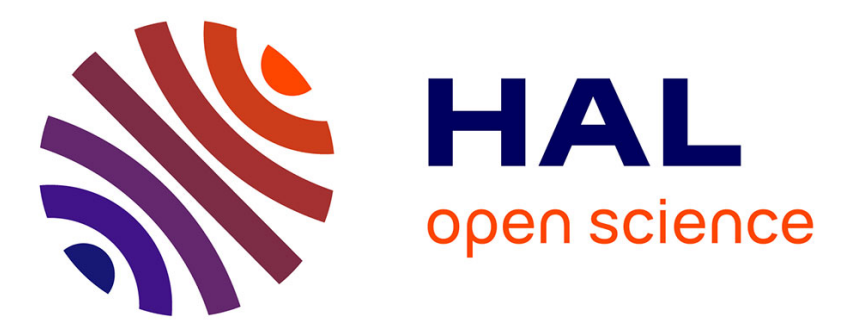

\title{
Virtual reconstruction of paranasal sinuses from CT data: A feasibility study for forensic application
}

P. Gach, L. Tuchtan-Torrents, C. Delteil, Pascal Adalian, M.D. Piercecchi, L. Ebert, G. Gorincour

\section{- To cite this version:}

P. Gach, L. Tuchtan-Torrents, C. Delteil, Pascal Adalian, M.D. Piercecchi, et al.. Virtual reconstruction of paranasal sinuses from CT data: A feasibility study for forensic application. Diagnostic and Interventional Imaging, 2018. hal-02047942

\section{HAL Id: hal-02047942 \\ https://hal-amu.archives-ouvertes.fr/hal-02047942}

Submitted on 22 Oct 2021

HAL is a multi-disciplinary open access archive for the deposit and dissemination of scientific research documents, whether they are published or not. The documents may come from teaching and research institutions in France or abroad, or from public or private research centers.
L'archive ouverte pluridisciplinaire HAL, est destinée au dépôt et à la diffusion de documents scientifiques de niveau recherche, publiés ou non, émanant des établissements d'enseignement et de recherche français ou étrangers, des laboratoires publics ou privés.

\section{(ㄷ)(1) $\$$}

Distributed under a Creative Commons Attribution - NonCommercial| 4.0 International 


\section{Virtual reconstruction of paranasal sinuses from CT data: a feasibility study for forensic application}

GACH P (1)*, TUCHTAN-TORRENTS L (2), DELTEIL C (2), ADALIAN P (2), PIERCECCHI MD (2), EBERT LC (3), GORINCOUR G (1)

(1) LiiE, EA 4264, CERIMED, Aix-Marseille University, 13005 Marseille, France

(2) ADES, UMR 7268, Aix-Marseille University, Block A, 13344 Marseille, France

(3) University of Zurich, Institute of Forensic Medicine Zurich, $\mathrm{CH}-8057$ Zurich,

Switzerland

*Corresponding author: Pierre Gach, LiiE, EA 4264, CERIMED, Aix-Marseille University, , 13005 Marseille, France.

E-mail: pierre.gach@gmail.com 


\section{Abstract}

Purpose: The purpose of this study was to report the feasibility of computed modelization and reconstitution of the paranasal sinuses, before and after trauma, from CT data.

Materials and methods: We modeled and reconstructed the paranasal sinuses of two patients, before and after trauma, using two different softwares (3DSlicer and Blender). Both patients had different numbers and locations of fractures. The 3DSlicer® software was used to create a 3D model from CT data. We then imported the 3D data into the Blender ${ }^{\circledR}$ software, to reconstruct and compare the dimensions of the paranasal sinuses before and after trauma.

Results: The 3 fragments of patient A and the 7 fragments of patient B could be repositioned in the pre-traumatic configuration. Distance measurements proved to be similar between pre and post-traumatic 3D volumes.

Conclusion: After simple trauma, bone facial anatomy reconstruction is manually feasible. The whole procedure could benefit from automatization through machine learning. However, this feasibility must be confirmed on more severely fractured paranasal sinuses, to consider an application in forensic identification.

Keywords: Paranasal sinuses; Modelization; Reconstitution; Facial trauma; Computed tomography $(\mathrm{CT})$

\section{Introduction}

Postmortem identification of deceased victims is a major forensic issue for which medical imaging plays a major role [1-4]. Indeed, in addition to genetic tests, fingerprint, and dental analyses, the comparison of ante and post-mortem imaging data appears promising, particularly the analysis of the paranasal sinuses.

Several studies have shown the relevance of identification of victims by recognition techniques using pre-mortem and post-mortem computed tomography (CT) data of paranasal sinuses (e.g. frontal, sphenoidal, ethmoidal and maxillary sinuses). This can be achieved through visual comparison by matching CT data of paranasal sinuses of an unidentified deceased victim with pre-mortem CT data of paranasal sinuses from any subject suspected to be the deceased victim [5-16]. 
However, as a limitation, a number of victims who could benefit from this technique have severe and distorting craniofacial trauma that could hamper this comparative analysis. For instance, in case of mass disaster such as an earthquake or an aircraft crash, the visual comparison of pre and post-mortem CT data could not be achieved due to the victims' shattered facial bones. To address this limitation, we hypothesized that advances in the analysis and post-processing of CT data could allow a virtual reconstruction of facial bone anatomy when it is modified by a trauma.

The purpose of this study was to report the feasibility of computed modelization and reconstitution of the paranasal sinuses, before and after trauma, from CT data.

\section{Materials and methods}

\section{Patients}

We retrieved the CT data of two patients' skulls from the digital archiving network of our institution, choosing cases where pre- and post-trauma acquisitions were available, with different numbers and locations of fractures. We anonymized and labelled them "patient A" and "patient B" (Fig. 1). Patient A was a 45-year-old man with 3 fragments, involving a piece of the lateral wall of the right maxillary sinus and two parts of the right zygomatic arch. Patient B was a 59-year-old man with 7 fragments, involving both maxillary sinuses, the nasal septum and the right frontal sinus.

\section{Modelization steps}

For each of the four CT acquisitions, we successively performed the following steps of analysis. We imported DICOM CT data from the skull (in a "soft" filter) on an open source software, 3DSlicer®, developed by the Laboratory of Mathematics in Imaging of the Harvard Medical School. We then used the "Crop Volume" module of this software to focus the volume of interest on the paranasal sinuses and the mastoids, in the transverse, sagittal and coronal planes, after having modeled the cranial volume in three dimensions (3D), using the volume rendering application. We used the "threshold effect" function of the "Editor" module to semi-automatically generate a highlighting of bone walls Then to refine the modeling manually, we used the "Paint Effect" function of the same module (Fig. 2A). The brush diameter of this function is customizable, ranging from a decimeter to a quarter of a millimeter, which allows precise modeling of both the thickest structures (e.g. the cranial 
vault) and the finest partitions (especially the walls of the ethmoidal cells). In the case of fractured volumes, we modeled each fragment with a different color, randomly chosen. This part required technical skills to wield the software's functions, and radiological skills to determine which bone was broken and to isolate each fragment from the others. Once highlighting was performed, we used the "Per-Structure Volumes", "Split Merge Volume" and then "Merge And Build" tabs of the "Editor" module, which generated three-dimensional volumes of the different fragments (Fig. 2B). We saved this 3D data as an ".stl" file, the standard format for 3D printing.

\section{Reconstitution steps}

The reconstruction part required the Blender ${ }^{\circledR}$ software, developed by the Blender Foundation. We started by importing 3D volumes from "pre-trauma" acquisitions into the Blender ${ }^{\circledR}$ workspace. The next step was to make the outlines of the volumes transparent. To do so, we clicked on the "Material" tab located in the right column and then on "+ New". We checked the "Transparency" box that appears in the drop-down menu below, then set the "Alpha" value of the "Z Transparency" submenu to 0.300. The last step was to click on the bottom menu tab that changes the way the 3D object is seen ("Viewport shading" menu), and to select "Material". This visualization mode makes it possible to measure distances inside the paranasal sinuses. To carry out these measurements, it was necessary to use the left column, to select the "Grease Pencil" tab, then to click on "Ruler / Protractor". This allows for distance or angulation measurements outside or inside the 3D volume, by rotating around, or zooming in or out (Fig. 2C). By pressing the "N" key, a drop-down menu appeared on the right of the screen, which allowed to change the scale of the 3D volume, or of the background.

The reconstitution of fragmented 3D volumes was achieved as follows: we imported on the same "3D scene" all the fragments, which positioned themselves in the same way as they were modeled on 3DSlicer®. The goal was then to reposition each fragment to reconstruct the paranasal sinuses. Fragments were selected with the right click, moved after pressing the "G" key, then dropped on the right position with the left mouse button (Fig. 2D). To change their orientation, we first changed the pivot point on the lower menu, choosing "3D Cursor". Then with the left mouse button we selected an adequate pivot point on the "3D scene". This pivot point was firstly used by pressing the "R" key, which enabled us to rotate the fragment. By secondarily pressing the "X", "Y" or "Z" keys, the rotation was done 
according to the 3 corresponding planes of the space, which made it possible to position the fragment in all the possible orientations. The fragment was then released with the left click.

\section{Modeling and reconstitution assessment}

Once the different fragments were repositioned, we made the volumes transparent according to the steps previously described. Then we performed different distance measurements (height, width, and depth) (Fig. 3). The height was considered the maximal craniocaudal diameter, the width the maximal transverse diameter and the depth of the maximal anteroposterior diameter $[17,18]$. The sphenoid sinuses were considered as a single entity, and the frontal sinuses and maxillary right and left sinuses were individualized [18]. Since patient A displayed a fracture of his right zygomatic arch (an element easily accessible to measurement), we measured the angle of this bone structure on both pre and post-fractured volumes.

\section{Results}

Patient A had facial bones fractures consecutive to a brawl. Patient B had Parkinson's disease and fell from his height. For both patients we were able to modelize CT data and reconstruct the traumatized facial anatomy in the same configuration as pre-traumatic CT. The various intra-sinus measurements of the two patients, including width, height and depth of each paranasal sinus are reported in Table 1. The dimensions of the different paranasal sinuses were similar between pre and post-fracture volumes, with a maximum error of $0.06 \mathrm{~mm}$. The inner angle of the pre-fractured right zygomatic arch of the patient A was $146^{\circ}$, vs. $143^{\circ}$ on the reconstructed post-fractured volume. The angle was measured in the middle of the arch (Fig. 4).

\section{Discussion}

In our study, softwares allowed a faithful modeling and reconstruction of the paranasal sinuses and the dimensions of the different paranasal sinuses were similar to those reported in the literature $[18,19]$. We used the soft kernel tissue patients' skulls data, considering that soft kernel reconstructions can be noticed on almost any head CT study, but the presence of hard kernel reconstructions could be inconstant [8].

To our knowledge, no similar study exists in the literature. In articles assessing identification by comparing CT data of paranasal sinuses, we found that authors compared pre 
and post-mortem CT data of paranasal sinuses by using reformatted axial images [9]. The data were considered matching when these reformatted images could be superposed. In our study we could not achieve to superpose the pre and post reconstituted 3D volume, so we opted to assess our reconstructions by measuring the maximum length, width and height of the different paranasal sinuses. Without comparison element, we considered a maximum error of $0.06 \mathrm{~mm}$ acceptable. The use of both free-access softwares was facilitated by consulting many written and video tutorials available on the internet. These programs run on standard computers, and do not require high-performance models dedicated to 3D modeling. The main disadvantage of the technique we describe lies in the time required for accurate modeling of the fragmented volumes, on the 3DSlicer® software. Artificial intelligence will probably help reduce this time.

Several studies have shown the relevance of identification by recognition, by matching post-mortem computed tomography (CT) data of paranasal sinuses (e.g. frontal, sphenoidal, ethmoidal and maxillary sinuses) of an unidentified deceased victim with older ("premortem") CT data of paranasal sinuses from a subject whose identity is supposed to be the right one [5-9]. Paranasal sinuses' anatomy is unique to each individual, even between homozygous twins, and remains stable during aging [6]. Its only possible variation is to thicken and / or partially shrink secondarily to certain local infections. It has significant resistance to direct trauma, and as a bone structure it resists the decomposition of soft tissue [8]. Regarding radiological identification methods, standard radiography has gradually given way to computed tomography by CT scan or cone-beam CT. The use of CT data appears more relevant than that of standard radiography because its analysis seems more objective, and due to its widespread use nowadays, in neurological and otorhinolaryngological conditions. On the other hand, the development of CB-CT in dental pre-implant assessments makes it possible to have a base of comparison more and more abundantly. Standard radiography also has the disadvantage of modifying the paranasal sinuses dimensions in terms of length / width / height according to the incidence of X-rays [11]. It is also possible to compare conventional pre-mortem standard radiography with projected images calculated from post-mortem $\mathrm{CT}$ data $[12,13]$. In addition to the increasing use of $\mathrm{CT}$ scan in the different fields of medicine, image archiving has even spread in forensic medicine departments, allowing to compare pre and post-mortem data [16].

We establish a first step, by showing in this feasibility study that a radiologist with basic skills in otorhinolaryngological anatomy can reconstitute the bone facial anatomy in simple 
traumatic cases. Further research could extend this reconstitution to more fragmented facial bones, in order to even consider forensic identification in the most traumatized cases. In these complex cases, mathematical approaches like statistical shape modeling should be tested [2022].

Our feasibility study has several limitations. We provided a single-observer study. Although the modelization required radiologic skills to be accurate, we considered that these little fractured paranasal sinuses would not be modelized or reconstituted differently by another radiologist. Indeed, each bone fragment of the post-fractured volumes was individualized from the others, minimizing the risk of error. Unlike authors who compared paranasal sinuses volumes [6], we could not achieve to measure volume of each of the paranasal sinuses with Blender®. Indeed, the 3D volumes are made of thousands of polygons, and we could not isolate the volume of the paranasal sinuses themselves from the total volume.

In conclusion, our results show that bone facial anatomy modelization and then reconstitution is manually feasible in poorly fractured paranasal sinuses cases, without requiring advanced skills or high-performance computers. The whole procedure could benefit from automatization through machine learning, especially considering the time required for the entire procedure. However, this feasibility must be confirmed on more severely fractured facial bones, to secondarily consider an application in forensic identification. In these complex cases, other approaches like statistical shape modeling should be tested.

\section{Conflict of interest}

The authors declare that they have no conflicts of interest concerning this article. 


\section{REFERENCES}

[1] Tuchtan L, Lesieur E, Bartoli C, Delteil C, Sarda-Quarello L, Torrents J, Sigaudy S, Piercecchi MD, Gorincour G, Diagnosis of congenital abnormalities with post-mortem ultrasound in perinatal death, Diagn Interv Imaging 99. (2018) 143-149.

[2] Tuchtan L, Gorincour G, Kolopp M, Massiani P, Léonetti G, Piercecchi-Marti MD, Bartoli C, Combined use of postmortem 3D computed tomography reconstructions and 3Ddesign software for postmortem ballistic analysis, Diagn Interv Imaging. 98 (2017) 809-812.

[3] Garetier M, Deloire L, Dédouit F, Dumousset E, Saccardy C, Ben Salem D, Postmortem computed tomography findings in suicide victims, Diagn Interv Imaging. 98 (2017) 101-112.

[4] Sifaoui I, Nedelcu C, Beltran G, Dupont V, Lebigot J, Gaudin A, Ridereau Zins C, Rouge Maillard C, Aubé C, Evaluation of unenhanced post-mortem computed tomography to detect chest injuries in violent death, Diagn Interv Imaging. 98 (2017) 393-400.

[5] Uthman AT, Al-Rawi NH, Al-Naaimi AS, Tawfeeq AS, Suhail EH, Evaluation of frontal sinus and skull measurements using spiral CT scanning: an aid in unknown person identification, Forensic Sci Int. 197 (2010) 124.e1-124.e7.

[6] Beaini TL, Duailibi-Neto EF, Chilvarquer I, Melani RF, Human identification through frontal sinus 3D superimposition: Pilot study with Cone Beam Computer Tomography, J Forensic Leg Med. 36 (2015) 63-69.

[7] Ruder TD, Kraehenbuehl M, Gotsmy WF, Mathier S, Ebert LC, Thali MJ, et al, Radiologic identification of disaster victims: A simple and reliable method using CT of the paranasal sinuses, Eur J Radiol. 81 (2012) e132-e138.

[8] Auffret M, Garetier M, Diallo I, Aho S, Ben Salem D, Contribution of the computed tomography of the anatomical aspects of the sphenoid sinuses to forensic identification, $\mathrm{J}$ Neuroradiol. 43 (2016) 404-414. 
[9] Brun CN, Christensen AM, Kravarski M, Gorincour G, Schweitzer W, Thali MJ, et al, Comparative radiologic identification with standardized single CT images of the paranasal sinuses: evaluation of inter-rater reliability, Forensic Sci Int.280 (2017) 81-86.

[10] Haglund WD, Fligner CL, Confirmation of human identification using computerized tomography (CT), J Forensic Sci. 38 (1993) 708-712.

[11] Quatrehomme G, Fronty P, Sapanet M, Grévin G, Bailet P, Ollier A, Identification by frontal sinus pattern in forensic anthropology, Forensic Sci Int. 83 (1996) 147-153.

[12] Pfaeffli M, Vock P, Dirnhofer R, Braun M, Bolliger SA, Thali MJ, Post-mortem radiological CT identification based on classical ante-mortem X-ray examinations, Forensic Sci Int. 171 (2007) 111-117.

[13] Soares CB, Almeida MS, Lopes Pde M, Beltrão RV, Pontual Ados A, Ramos-Perez FM, Figueroa JN, Pontual ML, Human identification study by means of frontal sinus imaginological aspects, Forensic Sci Int. 262 (2016) 183-189. [14] Souza LA Jr, Marana AN, Weber SAT, Automatic frontal sinus recognition in computed tomography images for person identification, Forensic Sci Int. 286 (2018) 252-264.

[15] Kim DI, Lee UY, Park SO, Kwak DS, Han SH, Identification using frontal sinus by three-dimensional reconstruction from computed tomography, J Forensic Sci. 58 (2013) 5-12. [16] Tatlisumak E, Yilmaz Ovali G, Aslan A, Asirdizer M, Zeyfeoglu Y, Tarhan S, Identification of unknown bodies by using CT images of frontal sinus, Forensic Sci Int. 166 (2007) 42-48. [17] Przystańska A, Kulczyk T, Rewekant A, Sroka A, Jończyk-Potoczna K, Lorkiewicz-Muszyńska D, Gawriołek K, Czajka-Jakubowska A, Introducing a simple method of maxillary sinus volume assessment based on linear dimensions, Ann Anat. 215 (2018) 4751. 
[18] Pirner S, Tingelhoff K, Wagner I, Westphal R, Rilk M, Wahl FM, Bootz F, Eichhorn KW, CT-based manual segmentation and evaluation of paranasal sinuses, Eur Arch Otorhinolaryngol. 266 (2009) 507-518.

[19] Sahlstrand-Johnson P, Jannert M, Strömbeck A, Abul-Kasim K, Computed tomography measurements of different dimensions of maxillary and frontal sinuses, BMC Med Imaging. $11(2011) 8$.

[20] Vlachopoulos L, Lüthi M, Carrillo F, Gerber C, Székely G, Fürnstahl P, Restoration of the patient-specific anatomy of the proximal and distal parts of the humerus: statistical shape modeling versus contralateral registration method, J Bone Joint Surg Am. 100 (2018) e50.

[21] Sinha A, Leonard S, Reiter A, Ishii M, Taylor RH, Hager GD, Automatic segmentation and statistical shape modeling of the paranasal sinuses to estimate natural variations, Proc SPIE Int Soc Opt En. (2016) 9784.

[22] Claes P, Vandermeulen D, De Greef S, Willems G, Suetens P, Craniofacial reconstruction using a combined statistical model of face shape and soft tissue depths: methodology and validation, Forensic Sci Int. 159 (2006) S147-S158. 


\section{Figures captions}

Figure 1: Axial images from CT data of pre and post-fractured paranasal sinuses from patients A and B. Pre-fractured paranasal sinuses of patient A appears on Fig. A, and pre-fractured paranasal sinuses of patient B on Fig. C. Bone fractures are rimmed in red. Patient A presents a fracture of the lateral wall of the right maxillary sinus (Fig. B). Patient B presents fractures of both maxillary sinuses, involving the right anterior wall and the left anterior and posterior walls (Fig. D).

Figure 2: A) Semi-automatically generated highlighting of paranasal sinuses' bone walls of Patient A pre-fractured volume in 3D Slicer $\AA$. The bone structures are highlighted in yellow in the 3 views (axial, sagittal and coronal) of the CT data of the head. B) 3D volume of Patient B post-fractured paranasal sinuses in 3D Slicer $\circledast$. The structure in yellow is the non-fractured volume. The element in dark green is the fractured anterior wall of the right maxillary sinus and the elements in light green and red are the bone pieces of the fractured nasal septum. C) Blender ${ }^{\circledR}$ interface - pre-fractured 3D volume of Patient A paranasal sinuses. The semitransparent walls allow distance measurements within the paranasal sinuses. D) 3D bone fragments of paranasal sinuses from Patient B. All of the seven bone fragments are shown next to the facial skeleton.

Figure 3: Example measurements of the Patient B paranasal sinuses dimensions. The white lines represent measurements of distances of maximum height, width and depth within the paranasal sinuses, whose walls appear semi-transparent thanks to the Blender® software.

Figure 4: Measurements of Patient A right zygomatic arch on pre (A) and post (B) fractured volumes.

Table 1: Dimensions of 3D reconstituted paranasal sinuses. 


\section{Patient B}

7 fragments

Pre-fracture

Post-fracture

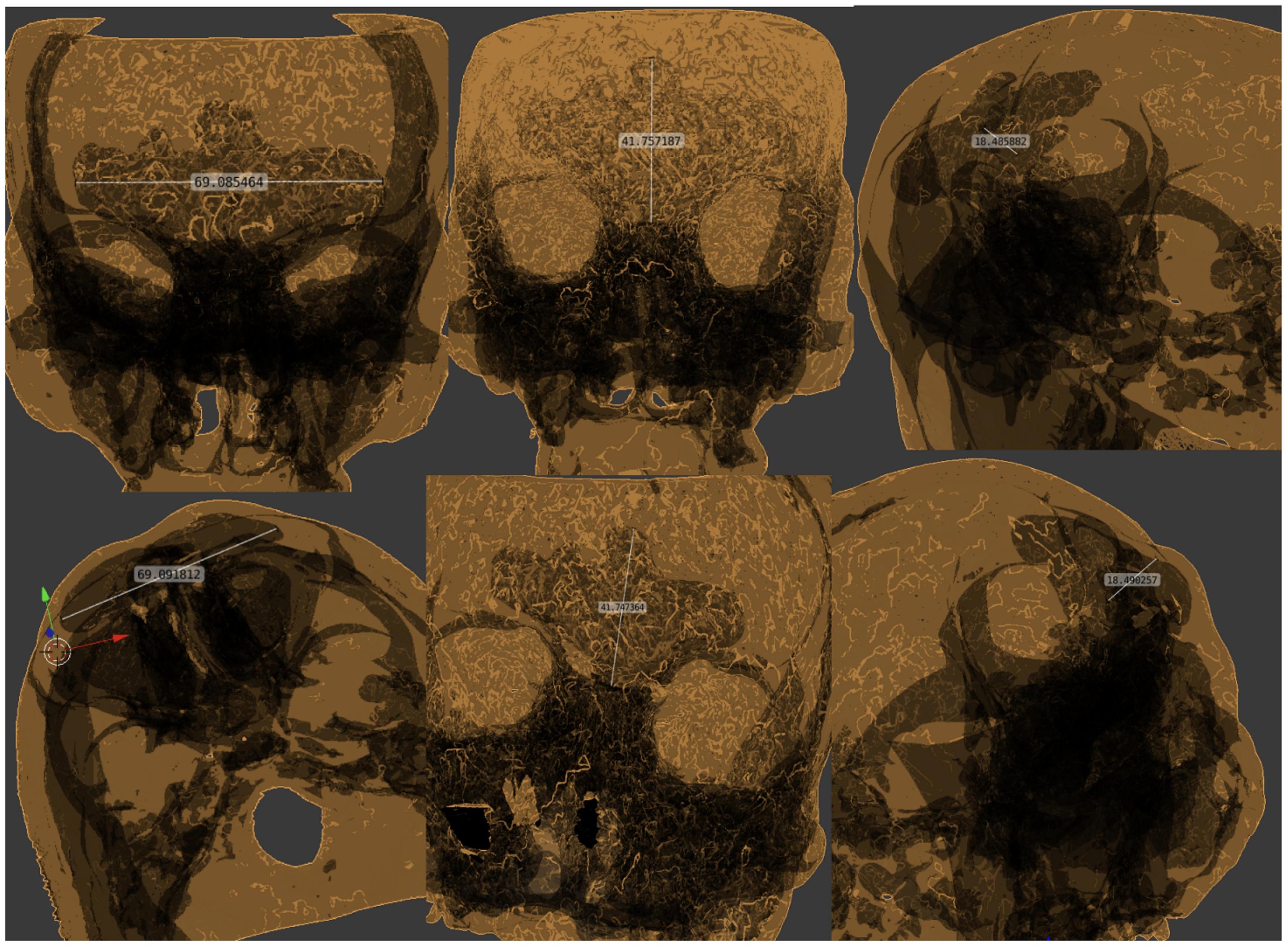




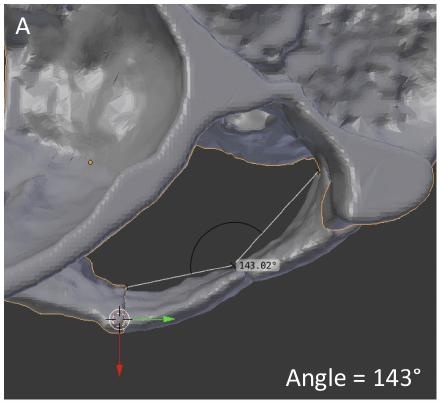


B

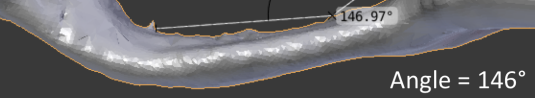




\begin{tabular}{rccc|ccc}
\hline & \multicolumn{3}{c|}{ Pre-trauma } & \multicolumn{3}{c}{ Post-trauma } \\
& Height & Width & Depth & Height & Width & Depth \\
\hline Patient A & & & & & & \\
Maxillary right & 31.85 & 26.04 & 40.11 & 31.87 & 26.06 & 40.11 \\
Maxillary left & 35.67 & 33.72 & 39.87 & 35.66 & 33.72 & 39.85 \\
Frontal & 41.04 & 70.09 & 22.53 & 41.02 & 70.10 & 22.51 \\
Sphenoidal & 29.07 & 30.82 & 27.21 & 29.05 & 30.83 & 27.21 \\
Patient B & & & & & & \\
Maxillary right & 34.26 & 27.02 & 36.78 & 34.26 & 27.01 & 36.77 \\
Maxillary left & 34.31 & 24.00 & 38.07 & 34.30 & 24.01 & 38.09 \\
Frontal & 41.76 & 69.08 & 18.48 & 41.74 & 69.09 & 18.49 \\
Sphenoidal & 29.16 & 30.85 & 27.26 & 29.16 & 30.91 & 27.27 \\
\hline
\end{tabular}

Note. Data are expressed in $\mathrm{mm}$ 